\title{
Tumores no germinales inusuales del testículo: tres casos clínicos
}

\author{
Van Cauwelaert Rojas R*, Ruiz-Tagle Phillips D*, Meneses Ciuffardi M**, \\ Carrasco Troncoso AM**, Aguirre Aguirre $\mathrm{C}^{*}$. \\ *Unidad de Urología, Fundación Arturo López Pérez, Santiago, Chile. **Unidad de Anatomía Patológica, \\ Fundación Arturo López Pérez, Santiago, Chile.
}

Actas Urol Esp. 2007;30(8):923-927

\section{RESUMEN}

TUMORES NO GERMINALES INUSUALES DEL TESTÍCULO: TRES CASOS CLÍNICOS

A propósito de tres casos clínicos con tumores de histología infrecuente del testículo que incluyen el adenoma de la rete testis, el tumor indiferenciado de los cordones sexuales y el mesotelioma de la túnica vaginal, se realiza una revisión bibliográfica de los tumores inusuales del testículo y sus anexos, incluyendo su incidencia y manejo.

Finalmente y a manera de conclusión, se explica la importancia de la biopsia rápida en el caso de los tumores testiculares dado que, aunque infrecuente, si ésta resultara benigna, podría evitarse la orquectomía radical y realizar una cirugia conservadora.

Palabras clave: Neoplasia testicular. Tumor de rete testis. Tumor estromal del cordón sexual. Mesotelioma.

\section{ABSTRACT}

THREE CASES OF UNUSUAL NON-GERM CELL TUMORS OF THE TESTICLE

By describing 3 clinical cases of unusual testicular non germinal tumors, including an adenoma of the rete testis, an undifferenciated sex cord tumor and a mesothelioma of the tunica vaginalis, we make a literature review of the unusual testicular tumors and testicular apendix, including their incidence and management.

Also and as one of our conclusions, we expose the importance of the intraoperatory biopsy in the testicular cancer surgery, because even if it is infrecuent, the presence of this rare testicular tumors, in which if they are proven to be benign, the testicular unit could be preserved and the radical orquiectomy could be avoided.

Keywords: Testicular neoplasms. Tumor of rete testis. Sex cord stromal tumors. Mesothelioma.

$\mathrm{L}$ a presencia o diagnóstico de tumor testicular tiene un gran impacto ya que afecta fundamentalmente a la población joven ${ }^{1,2}$. Los cánceres testiculares tienen una incidencia mundial de 2,05 por 100.000 varones y en Chile alrededor de 3,5 por 100.000 varones $^{2}$. El 95\% son tumores derivados del parénquima (línea germinal), seminoma y no seminoma. El resto corresponde a tumores de la línea no germinal (Leydig, Sertoli, Gonadoblastoma, entre otros). La clasificación para este tipo de tumores utilizada por nuestros patólogos es la recomendada por la OMS (Tabla 1$)^{3}$.

A propósito de 3 casos clínicos, se decide la revisión bibliográfica de estas histologías poco frecuentes del testículo y sus anexos.

\section{CASOS CLÍNICOS}

\section{Caso 1}

Hombre de 36 años, con diagnóstico de varicocele izquierdo, es sometido a cirugía en otro centro, encontrándose nódulo de la túnica vaginal, el cual se reseca y se envía a biopsia diferida, informándose la existencia de un mesotelioma con lesión focal en un borde de sección. Ocho meses después consulta en nuestro servicio, evaluándose con imágenes que resultan normales. Se efectúa exploración genital, resecándose por completo la túnica vaginal. La biopsia demuestra márgenes negativos y un foco microscópico alejado de éstos últimos. Hasta la actualidad los controles no han mostrado recidivas.

\section{Histología}

Tejido denso, con proliferación de estructuras glandulares. Las estructuras son de pequeño tamaño con epitelio cuboide. El estroma es desmoplásico, con proliferación focal de células fusadas. Varias de las estructuras glandulares presentan cuerpos de psammoma. La inmunohistoquímica es positiva para pancitoqueratina, calretini- 
Tabla 1. Tumores no germinales del testículo

\section{Formas Puras}

- Tumor de células de Leydig

- Tumor de células de Leydig maligno

- Tumor de células de Sertoli

- Variante rica en lípidos

- Variante Esclerosante

- Variante de Células Grandes calcificante

- Tumor de Sertoli maligno

- Tumor de células de la Granulosa

- Tipo adulto

- Tipo juvenil

- Tumores del tipo Tecoma/Fibroma

- Tecoma

- Fibroma

- Tumor de los cordones sexuales/estroma gonadal incompletamente diferenciado

- Tumor de los cordones sexuales/estroma gonadal formas mixtas

- Tumores malignos de los cordones sexuales/estroma gonadal

- Tumores que contienen tanto células germinales y células de los cordones sexuales/estroma gonadal - Gonadoblastoma

- Tumor inclasificable de células germinales y cordones sexuales/estroma gonadal

- Tumores misceláneos del testículo

- Carcinoide

- Cistoadenoma mucinoso

- Cistoadenocarcinoma

- Neuroblastoma

- Otros

- Tumores Hematopoyéticos

- Tumores de los Conductos y Rete Testis

- Adenoma

- Carcinoma

- Tumores de Estructuras Paratesticulares

- Tumor adenomatoide

- Mesotelioma Maligno

- Mesotelioma benigno

- Mesotelioma papilar bien diferenciado

- Mesotelioma quístico

- Adenocarcinoma de epidídimo

- Otros

- Tumores Mesenquimáticos del cordón y Anexos

na, citoqueratina 5/6, HBME-1 y vimentina. Es negativo para antígeno carcinoembrionario, CD99 y CD15. (Figs. 1, 2 y 3). Conclusión: "Mesotelioma epitelioide, con componente de células fusadas, maligno, de bajo grado".

\section{Caso 2}

Paciente de 22 años consulta por hallazgo de tumor en ecotomografía escrotal, con marcadores tumorales con valores normales y estudio de imágenes negativos para diseminación. Esta lesión se aprecia rodeada por una membrana en toda su extensión. Se efectúa exploración genital y por la sospecha de un posible tumor no germinal, se realiza biopsia rápida, que no es concluyente por lo que se efectúa una orquiectomía ampliada.

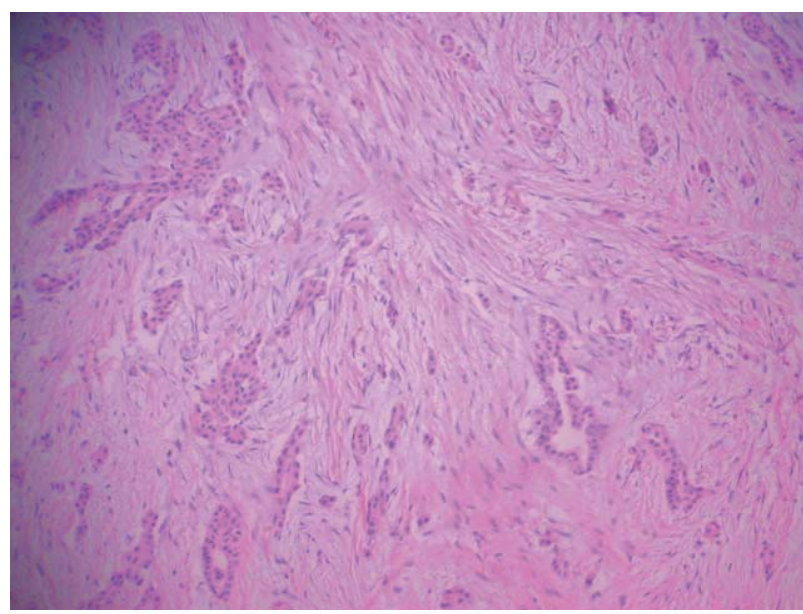

\section{FIGURA 1}

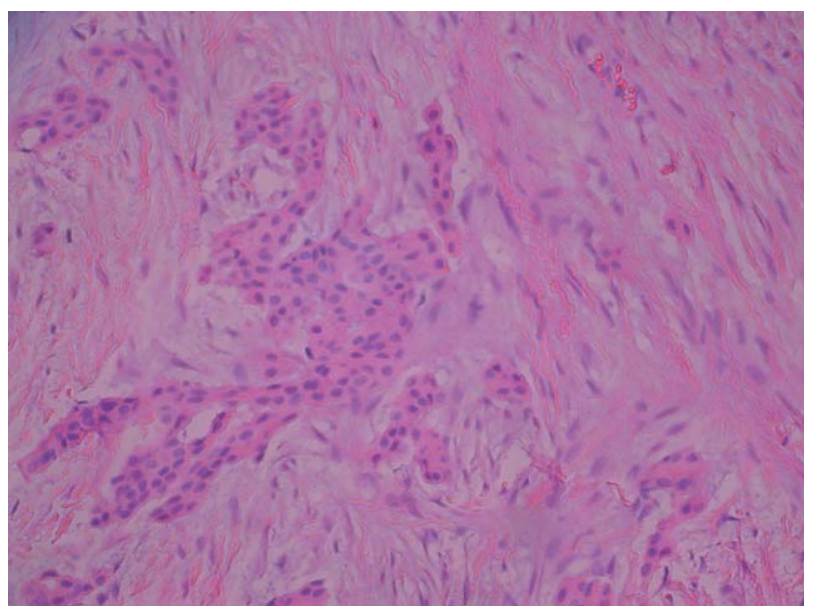

FIGURA 2

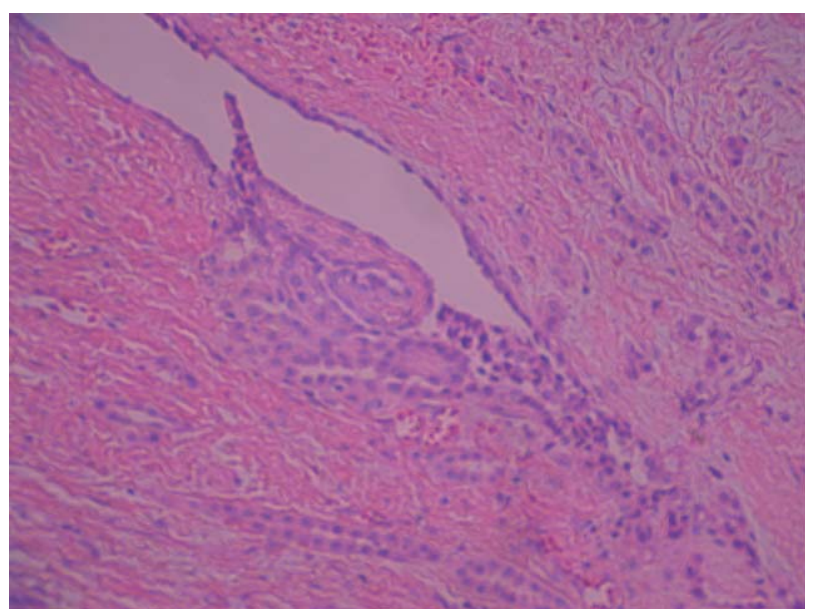

FIGURA 3 


\section{Histología}

La biopsia demuestra un tumor de color amarillo de 1,6 cm. de diámetro, cuyo informe histológico describe una neoplasia inclasificable de cordones sexuales y estroma gonadal, con los caracteres de tumor de células de la granulosa y áreas de aspecto fusado predominante positivo para inhibina. Existe diferenciación focal epitelioide con formación rudimentaria de túbulos. En otros sitios, existen túbulos seminíferos atrapados que se destacan con la tinción de fosfatasa alcalina placentaria (PLAP). (Figuras 4, 5 y 6). Conclusión: "Tumor estromal no clasificable de cordones sexuales/estroma gonadal con neoplasia intratubular de células germinales en los túmulos seminíferos atrapados" Esta lesión inusual, se comparte con anatomía patológica de la Clínica Mayo que confirman el diagnóstico de nuestros patólogos.

\section{Caso 3}

Paciente de 36 años, sometido a orquiectomía izquierda por atrofia testicular en otro centro; la biopsia es informada como adenocarcinoma de la rete testis y derivado a nuestra institución. El paciente presenta marcadores tumorales negativos y tomografia abdominal sin signos de diseminación.

\section{Histología}

El informe anatomopatológico describe una combinación entre atrofia e hiperplasia de la rete testis; las zonas hiperplásicas muestran células columnares formando espacios de arquitectura compleja pero sin actividad mitótica, atipia o crecimiento sólido o cribiforme significativo. A pesar de su arquitectura compleja, no hay evidencia de comportamiento invasivo. (Figuras 7, 8 y 9). Conclusión: "Atrofia testicular e hipoespermatogénesis asociado a hiperplasia adenomatosa de la rete testis"; una vez efectuada la revisión por nuestros patólogos, la biopsia fue confirmada por el grupo de patólogos de la Clínica Mayo, que sugieren al igual que los nuestros un comportamiento benigno de dicha histología.

\section{DISCUSIÓN Y CONCLUSIONES}

Urólogos, radioterapeutas y quimioterapeutas saben perfectamente que hacer frente a los seminomas y no seminomas; sin embargo, las conductas no son uniformes cuando se enfrentan tumores poco frecuentes de la línea no germinal y donde se discute muchas veces su potencial maligno. Esta situación puede condicionar tratamientos

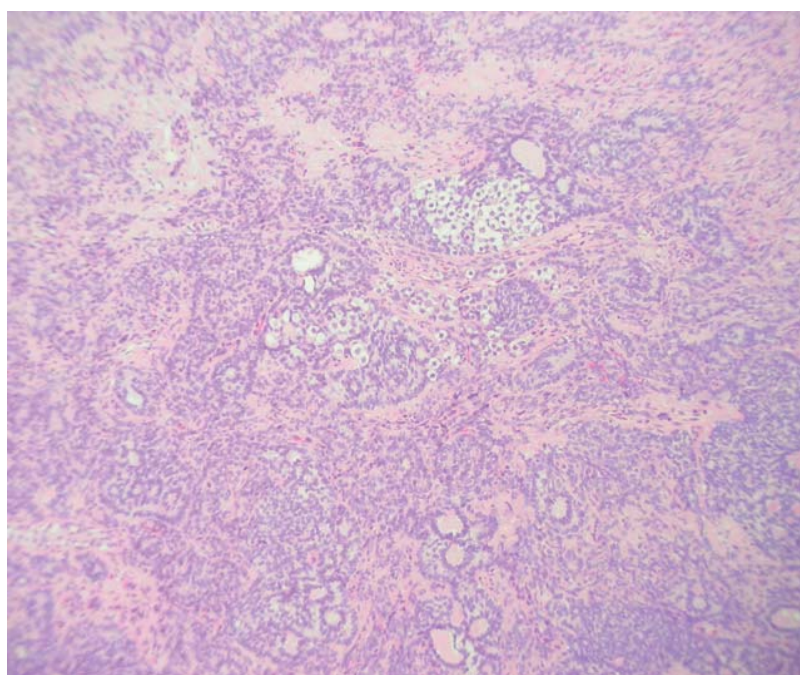

FIGURA 4

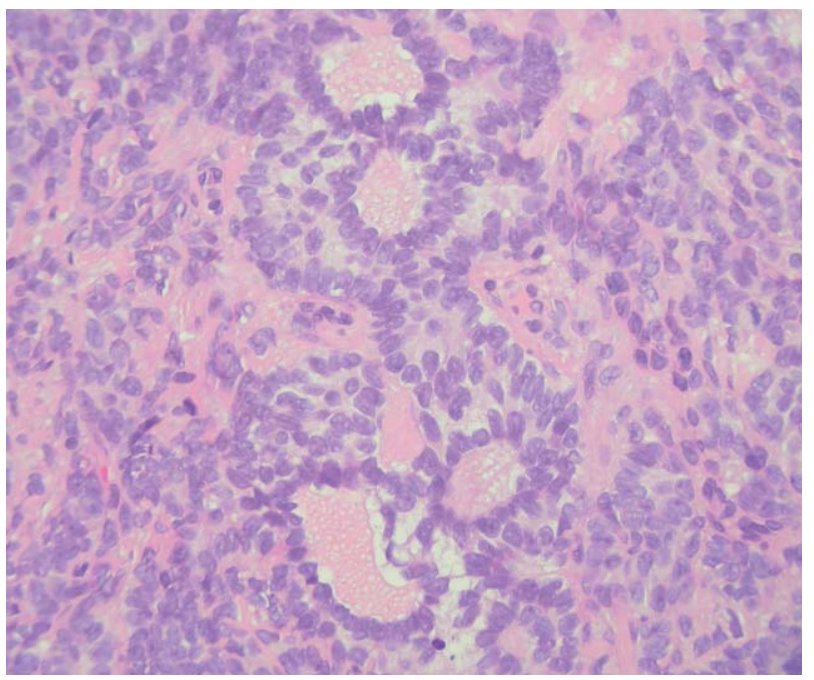

FIGURA 5

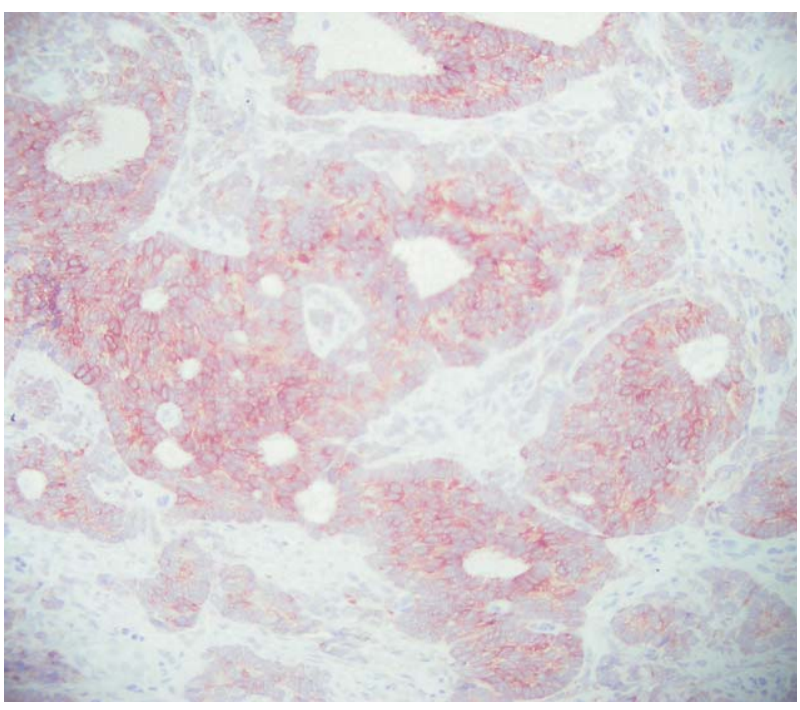

FIGURA 6 
radicales innecesarios ${ }^{4}$ o no adoptar una conducta agresiva necesaria. La posibilidad de encontrar un mayor número de lesiones testiculares benignas y tumores no germinales son cada vez más frecuentes con el uso rutinario de la ecotomografía escrotal $^{5}$. Una mejor precisión hoy día en el diagnóstico de estos tumores no germinales, ha permitido según la clasificación de la OMS asegurar en cada uno de ellos su potencial maligno (0-benigno a 4maligno) y con ello ayudar a tomar las decisiones conservadoras o más agresivas dependiendo de esta información. Esto hace necesario la presencia del patólogo para el análisis de biopsia rápida, cuando se sospecha que estamos frente a la presencia de un tumor no germinal.

Los tumores intraescrotales no testiculares pueden derivar del epidídimo, del cordón espermático, tejido escrotal (adiposo, conectivo, muscular, etc.), de la túnica vaginal o ser metastásicos ${ }^{6}$.

Las lesiones que se originan en estas estructuras pueden ser quísticas o sólidas; en las quísticas prácticamente se puede asegurar su benignidad, no así las sólidas. Estas lesiones en un $3 \%{ }^{7}$. son malignas, lo que refuerza efectuar biopsia rápida frente a estas lesiones intraescrotales, pudiendo de esta manera evitar cirugías radicales o apoyarlas en caso de malignidad.

El mesotelioma maligno es un tumor que se origina de la vaginal o la túnica albugínea y se considera un tumor raro, a pesar de ser la neoplasia paratesticular más frecuente después del sarcoma. Hasta el año 2002, hay descritos en la literatura sólo alrededor de 80 casos. La edad media de presentación es de 55 años, y su único factor de riesgo conocido es la exposición al asbesto.

La histología habitualmente describe una morfología papilar y tubulopapilar, con áreas sólidas celulares. La estructura celular puede ser variable, variando entre redondeada o cuboideas y aplastadas o columnares. Hay variación del tamaño celular y forma. El citoplasma es eosinofílico y tienen nucleolo prominente.

La inmunohistoquímica se muestra positiva con citoqueratina, vimentina y calretinina, como ocurre en el caso descrito. Si bien es cierto que este tumor tiene un potencial de bajo grado de malignidad, en nuestro caso la conducta fue conservadora ya que había transcurrido varios meses entre la primera y segunda cirugía.

Los tumores testiculares de los cordones sexuales son entidades raras. Mostofi ${ }^{8}$ y posterior- mente Young ${ }^{9}$ clasificaron los tumores de células de Leydig, Sertoli y de Sertoli-Leydig, además de los tumores de la granulosa y los mixtos. Dentro de los tumores de Sertoli existen 3 tipos, el clásico tiene preferentemente presentación en pacientes pediátricos ${ }^{10}$; los de células grandes calcificadas, suelen asociarse a acromegalia, gigantismo hipofisiario, muerte súbita, pigmentación mucocutánea y el sindrome de Peutz-Jeghers ${ }^{10}$. La tercera variedad de tumores de Sertoli, esclerosante, que presenta abundante fibrosis y hialinización. De éste tipo hay descritos sólo 13 casos en el mundo ${ }^{11,12}$.

Los tumores de Sertoli son los más frecuentes de la línea no germinativa y de ellos el 10\% son malignos. Nuevamente, la biopsia rápida puede evitar la extirpación de la gónada.

Los tumores de la granulosa es un grupo heterogéneo de tumores testiculares, que han sido descritos bajo un sinnúmero de nombres pero que no son clasificables dentro de otro grupo más específico. Están compuestos mayoritariamente por tejido indiferenciado, con formación tubular rudimentaria, islas de células de Leydig u otras estructuras.

Aunque heterogéneo, muchos de estos tumores son similares y están conformados en su mayoría por células fusadas pequeñas y redondeadas, con depresiones en el núcleo y componente epitelioide escaso. A la inmunohistoquímica, estos tumores son habitualmente reactivos a la actina del músculo liso y a la proteína S-100, al igual que los tumores de la granulosa, pero a diferencia de éstos, son generalmente queratina negativos.

A pesar de los pocos casos descritos en la literatura, sólo se ha comunicado uno de comportamiento maligno, por lo que el manejo en este paciente ha sido conservador luego de la orquiectomía, manteniéndose en observación.

$\mathrm{El}$ adenoma de la rete testis es otro tumor no germinal, que ocurre en asociación a varias anormalidades testiculares; la más frecuente es la atrofia testicular ${ }^{13}$. Esta hipertrofia adenomatosa ha sido reportada en pacientes con criptorquidea, insuficiencia hepática, displasia renal bilateral y en asociación a tumores de células germinales. El diagnóstico diferencial con el adenocarcinoma de la rete testis es histológico, el cual presenta patrones histológicos diferentes y con un pronóstico de sobrevida descrito entre 8 meses y 4 años.

$\mathrm{El}$ adenoma de la rete testis es un tumor epitelial raro que se presenta en una rete testis dilatada y habitualmente tiene un patrón tubular 


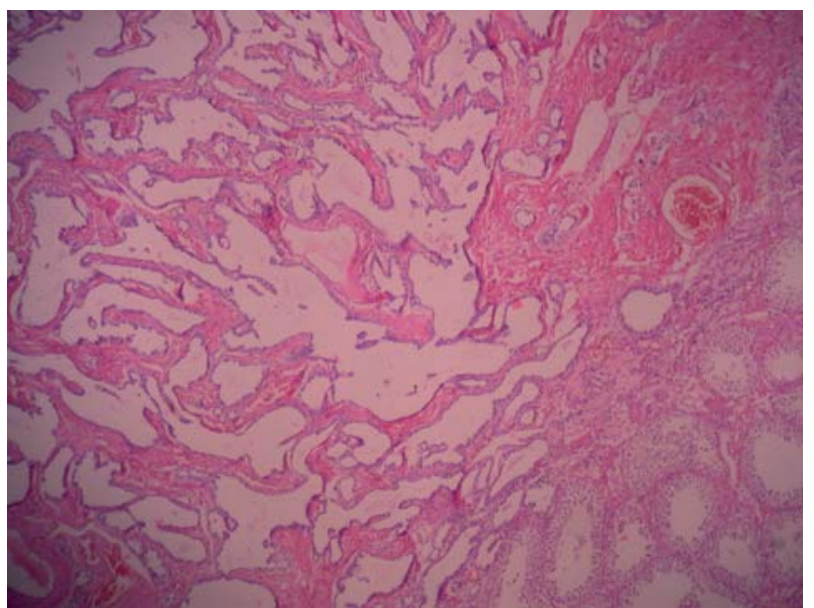

FIGURA 7

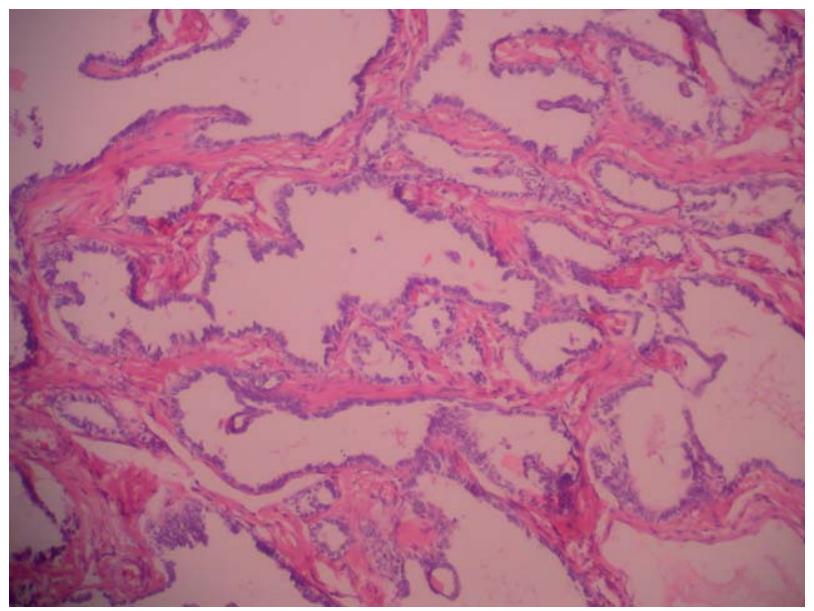

FIGURA 8

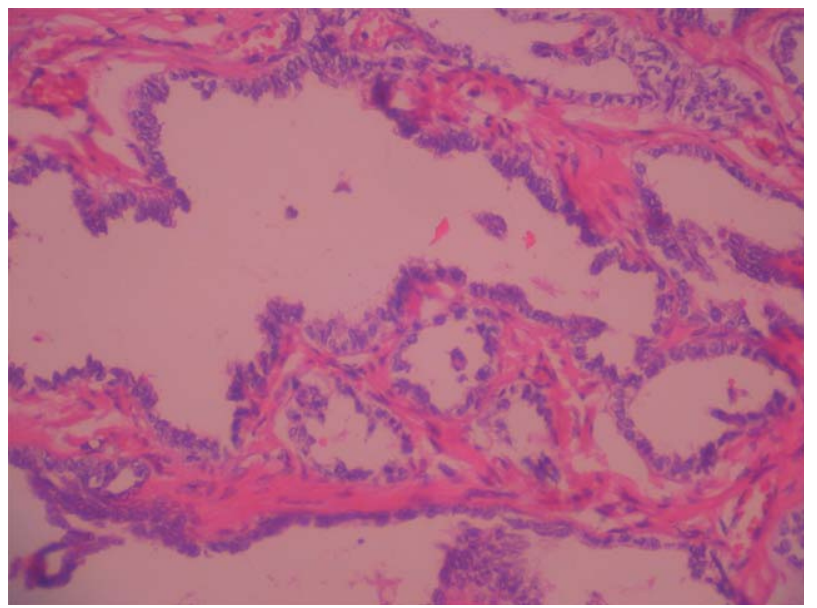

FIGURA 9

que se asemeja al tumor de células de Sertoli. Éste es un tumor raro que ocurre fundamentalmente en adultos, formando nódulos polipoideos compuestos por túbulos, que se proyectan hacia el lumen de la rete testis ${ }^{14,15}$.
Finalmente, la mejoría en las técnicas de microscopía de luz, la mejor clasificación de estos tumores y el advenimiento de la inmunohistoquímica, permite con mucha seguridad tomar decisiones correctas al momento de operar estos pacientes, permitiendo la conservación de la glándula en numerosos casos, la extracción de ésta en los dudosos y el tomar conductas agresivas en el postoperatorio con los antecedentes anatomopatológicos y el resultado de la inmunohistoquímica.

Agradecimientos: Se agradece al Dr. Jeffrey L. Myers de la Clínica Mayo por la revisión de las piezas histológicas enviadas para comprobación.

\section{REFERENCIAS}

1. http://www.nci.nih.gov/espanol/pdq/tratamiento/testiculo/healthprofessional, 2004, "Cancer Testicular", National Cancer Institute.

2. http://www.colegiomedico.cl, Protocolo AUGE, 2004, "Cancer de Testículo y Células Germinales Extragonadales del Adulto", pag 3.

3. Eble J. Tumours of the Urinary System and Male Genital Organs, 2004, "Tumours of the Testis and paratesticular tissue" pag 217-278.

4. Srigley JR, Hartwick RW. Tumors and cysts of the paratesticular region. Pathol Annu. 1990;25(pt 2):51-108.

5. Carmignani L, Gadda F, Gazzano G, Nerva F, Mancini M, Ferruti M, et al. High incidence of benign testicular neoplasms diagnosed by ultrasaund. J Urol. 2003;170(5):1783-1786.

6. Ruiz JM; Ruiz J; Aguas C; Vaillo V; Gutiérrez A; García M. Proliferación nodular y difusa fibrosa de la túnica vaginal del testículo. Presentación de un nuevo caso y revisión de la literatura. Rev Esp Patol. 2004;37(1):91-98.

7. Beccia DJ, Krane RJ, Olsson CA. Clinical management of non-testicular intrascrotal tumors. J Urol. 1976;116:476-479.

8. Mostofi FK, Theiss EA, Ashley DJ. Tumors of specialaed gonadal stroma in human male subjects. Cancer. 1959;12: 944-957.

9. Young RH, Koelliker DD., Scully RE. Sertoli cel tumor of the testis, not otherwise specified. A clinicopathologic analysis of 60 cases. Am J Surg Pathol. 1998;22(6):709-721.

10. Gallego Sales S, Guerrero Medrano J. Tumor de células de Sértoli esclerosante. Presentación de un caso y revisión de la literatura. Rev Mex Urol. 2004;64(2):85-88.

11. Zukerberg LR, Young RH, Scully RE. Sclerosing Sertoli cell tumor of the testis. A report of 10 cases. Am J Surg Pathol. 1991;15(9):829-834.

12. Reale D, Pascale M, Virtullo G, Di Virgilio M, Pizzicanella J, Piccolotti G. Sclerosing sertoli cell tumor of the testis. Minerva Urol Nephrol. 2002;54(3): 179-182.

13. Amin MB. Selected other problematic testicular and paratesticular lesions: rete testis neoplasms and pseudotumors, mesothelial lesions and secondary tumors. Modern Pathol. 2005;18(Suppl 2):131-145.

14. Ulbright TM,Amin MB, Young RH. Tumors of the testis, adnexa, spermatic cord and scrotum, 3rd series, fascicle 25. Armed Forces Institute of Pathology: Washington DC, 1999.

15. Hartwick RW, Ro JY, Srigley JR, Ordoñez NG, Ayala AG. Adenomatous Hyperplasia of the rete testis. A clinicopathologic study of nine cases. Am J Surg Pathol. 1991;15(4): 350-357.

Correspondencia autor: Dr. D. Ruiz-Tagle Phillips

Unidad de Urología. Fundación Arturo López Pérez. Santiago, Chile. Tel.: 24252110

E-mail autor: danielrt@mi.cl

Información artículo: Nota clínica

Trabajo recibido: abril 2006

Trabajo aceptado: octubre 2006 